\title{
EFEITO DA OMISSÃO DE MACRONUTRIENTES E BORO NO CRESCIMENTO, NOS SINTOMAS DE DEFICIÊNCIAS NUTRICIONAIS E NA COMPOSIÇÃO MINERAL DE PLANTAS DE CAMUCAMUZEIRO ${ }^{1}$
}

\author{
ISMAEL DE JESUS MATOS VIÉGAS², MARIA ALICE ALVES THOMAZ³, JEFFERSON FELIPE DA SILVA ${ }^{4}$, HERÁCLITO \\ EUGÊNIO OLIVEIRA DA CONCEIÇÃO ${ }^{5}$, ANA PRISCILLA MIRANDA NAIFF ${ }^{6}$
}

\begin{abstract}
RESUMO - Com o objetivo de avaliar o efeito da omissão de macronutrientes e do micronutriente boro no crescimento, nos sintomas de deficiências nutricionais e na composição mineral em plantas de camucamuzeiro, conduziu-se experimento em casa de vegetação, mediante a técnica do elemento faltante. O delineamento experimental foi o inteiramente casualizado, com quatro repetições e oito tratamentos, sendo completo (N, P, K, Ca, Mg, $\mathrm{S}$ e micronutrientes) e omissão individual de N, P, K, Ca, Mg, S e B. Os sintomas visuais de deficiências foram, de modo geral, de fácil caracterização para todos os nutrientes. Com exceção do fósforo, as omissões dos demais nutrientes afetaram a produção de matéria seca, quando comparados ao tratamento completo. Com base nos teores em $\mathrm{g} \mathrm{kg}^{-1}$, dos macronutrientes, e em mg kg-1, do micronutriente boro nas folhas, infere-se em uma primeira aproximação dos valores adequados (completo), ou seja: 16,9 a 18,2 de N ; 1,2 a 1,9 de P; 5,2 a 6,0 de K; 9,9 a 11,7 de Ca; 1,4 a 3,6 de Mg; 2,4 a 2,8 de $\mathrm{S} ; 8,4$ a 9,5 de B e do deficiente (omissão), 6,5 a 7,9 de N; =0,9 de P; $=1,7$ de $\mathrm{K} ; 5,4$ a 6,5 de Ca; $=0,7$ de $\mathrm{Mg} ; 0,7$ a 1,2 de $\mathrm{S}$ e 1,1 a 1,9 de B Termos para indexação: fruticultura, crescimento, sintomas visuais, Myrciaria dúbia, nutrição.
\end{abstract}

\section{EFFECT OF OMISSION OF MACRONUTRIENT AND BORON ON GROWTH, ON SYMPTOMS OF NUTRITIONAL DEFICIENCY AND MINERAL COMPOSITION IN CAMUCAMUZEIRO PLANTS (Myrciaria dubia)}

\begin{abstract}
The effects of omission of macronutrient and boron on growth, on symptoms of nutritional deficiency and mineral composition of plants of "camucamuzeiro" were evaluated. The experiment was carried out in a greenhouse, by means of the missing element technique. The experimental design was completely randomized, with eight treatments and four repetitions, including complete ( $\mathrm{N}, \mathrm{P}, \mathrm{K}, \mathrm{Ca}, \mathrm{Mg}, \mathrm{S}$ and micronutrients) and individual omission of $\mathrm{N}, \mathrm{P}, \mathrm{K}, \mathrm{Ca}, \mathrm{Mg}, \mathrm{S}$ and $\mathrm{B}$. The visual symptoms of deficiency were easily characterized for all the nutrients. Excepting for $\mathrm{P}$, dry mass was affected by the omission of all the other nutrients, when compared with the complete treatment. Based on the macronutrient ( $\mathrm{g}$ kg$\left.{ }^{1}\right)$ and on the micronutrient boron $\left(\mathrm{mg} \mathrm{kg}^{-1}\right)$ on leaves contents, a first approach of the adequate values is inferred as being: $\mathrm{N}=16,9$ to 18,$2 ; \mathrm{P}=1,2$ to 1,$9 ; \mathrm{K}=5,2$ to 6,$0 ; \mathrm{C}=9,9$ to 11,$7 ; \mathrm{Mg}=1,4$ to 3,$6 ; \mathrm{S}=2,4$ to 2,$8 ;$ and $\mathrm{B}=8,4$ to 9,5 for the complete treatment, and $\mathrm{N}=6,5$ to 7,$9 ; \mathrm{P}=0,9 ; \mathrm{K}=1,7 ; \mathrm{Ca}=5,4$ to 6,$5 ; \mathrm{Mg}=0,7 ; \mathrm{S}=0,7$ to 1,2 and $\mathrm{B}=1,1$ to 1,9 for the deficient ones (omission).
\end{abstract}

Index terms: Myrciaria dubia, fruit crop, growth, visual symptoms, nutrition.

\section{INTRODUÇÃO}

O camucamuzeiro (Myrciaria dubia (H. B. K.) McVaugh) é uma planta tipicamente amazônica, pertencente à família myrtaceae. Apresenta porte arbustivo e vegeta espontaneamente às margens de praias, de rios, e dos lagos da Amazônia Brasileira, sendo encontrada na estação chuvosa, parcial ou totalmente submersa.

Sua importância socioeconômica reside no fato de seus frutos apresentarem alto teor de vitamina $\mathrm{C}$, em torno de $2.880 \mathrm{mg}^{100 \mathrm{~g}^{-1} \mathrm{de}}$

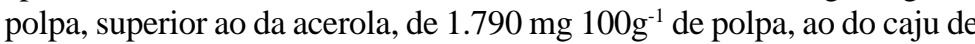

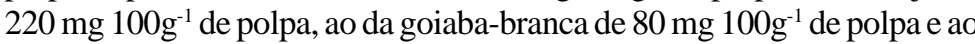

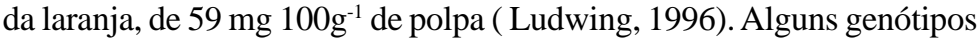

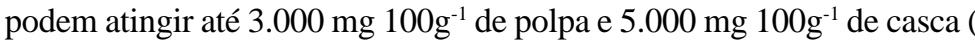
Ribeiro, 2002). Dada à elevada acidez, os frutos dificilmente são consumidos in natura. Na Amazônia Peruana, são utilizados para o preparo de refresco, sorvete, picolé, geléia, doce e licor; no Brasil, para o preparo de cosméticos e, artesanalmente, em sucos e licores, enquanto nos Estados Unidos, Japão e França, as indústrias farmacêuticas os transformam em tabletes de vitamina C.

Por não ser uma espécie domesticada, pouco se sabe sobre as condições ideais para o cultivo do camucamuzeiro, uma vez que há poucos trabalhos de pesquisa sobre essa espécie, principalmente na área de nutrição, podendo-se citar o de Yuyama et al.( 2003). Desse modo, tornase necessária a domesticação do camucamuzeiro, através do desenvolvimento de várias ações de pesquisas, entre as quais as de nutrição mineral.

O experimento foi instalado com o objetivo de avaliar o efeito da omissão de $\mathrm{N}, \mathrm{P}, \mathrm{K}, \mathrm{Ca}, \mathrm{Mg}, \mathrm{S}$ e B no crescimento, nos sintomas de deficiências nutricionais, assim como na composição mineral em plantas de camucamuzeiro.

\section{MATERIALEMÉTODOS}

O experimento foi conduzido durante 310 dias em condições de casa de vegetação da Embrapa Amazônia Oriental, Belém, Pará, com temperaturas variando de 25 a $30^{\circ} \mathrm{C}$. O delineamento experimental foi o inteiramente casualizado, com quatro repetições e oito tratamentos: completo (N, P, K, Ca, Mg, S e micronutrientes), omissão de um dos nutrientes: N, P, K, Ca, Mg, S e B.

Foram utilizadas sementes de germoplasma, coletadas na microrregião Alto Solimões, nos Municípios de São Paulo de Olivença e Atalaia do Norte, no Estado do Amazonas. A semeadura foi realizada em canteiro, contendo serragem fina bem curtida. Ao atingirem em torno de 10 $\mathrm{cm}$ de altura, as mudas foram repicadas para sacos de plástico preto em dimensões de $12 \mathrm{~cm}$ de largura x $25 \mathrm{~cm}$ de altura, contendo mistura de terra preta e serragem na proporção de 3:1. Quando as plantas atingiram aproximadamente $35 \mathrm{~cm}$ de altura, foram selecionadas, suas raízes lavadas com água corrente e, posteriormente, com água destilada, para eliminar possíveis resíduos do substrato e transplantadas para os vasos. Inicialmente, as plantas foram aclimatadas por um período de aproximadamente 60 dias, em solução nutritiva de Bolle-Jones (1954), modificada e diluída de 1:10, tendo, como fontes, sais P.A. A Tabela 1 apresenta a composição química da solução nutritiva, utilizada durante a condução do experimento. As plantas, ao atingirem altura média de $50 \mathrm{~cm}$,

\footnotetext{
${ }^{1}$ (Trabalho 124/2003). Recebido: 01/09/2003. Aceito para publicação: 08/07/2004.

${ }^{2}$ Eng. Agrôn., D.Sc., Pesquisador da Embrapa Amazônia Oriental e Professor Visitante da Universidade Federal Rural da Amazônia, Caixa Postal 48, CEP 66017- 970, Belém, Pará, e-mail: ismael@cpatu.embrapa.br

${ }^{3}$ Eng. Agrôn., M.Sc., Técnica da Fundação de Parques e Áreas Verdes de Belém, Funverde, e-mail: Alicethomaz@ aol.com

${ }^{4}$ Eng. Agrôn., Pesquisador da Embrapa Amazônia Oriental, e-mail:jefferson@cpatu.embrapa.br

${ }^{5}$ Eng. Agrôn., D.Sc. Pesquisador da Embrapa Amazônia Oriental: heraclit@ cpatu.embrapa.br

${ }^{6}$ Estudante de agronomia da Universidade Federal Rural da Amazônia, Caixa Postal 917, CEP 66095-100, Belém-PA, e-mail:apnaiff@ hotmail.com.
} 
TABELA 1 - Composição química da solução nutritiva (ml/L) de Bolle-Jones (1954) modificada, utilizada no experimento.

\begin{tabular}{|c|c|c|c|c|c|c|c|c|c|}
\hline \multirow[b]{2}{*}{ Solução-estoque } & \multicolumn{9}{|c|}{ Tratamentos } \\
\hline & Conc. & Completo & $-\mathrm{N}$ & $-\mathrm{P}$ & $-K$ & $-\mathrm{Ca}$ & $-\mathrm{Mg}$ & $-\mathrm{S}$ & $-\mathrm{B}$ \\
\hline $\mathrm{NaH}_{2} \mathrm{O}_{4}$ & $\mathrm{M}$ & 1 & 1 & - & 1 & 1 & 1 & 1 & 1 \\
\hline $\mathrm{Ca}\left(\mathrm{NO}_{3}\right)_{2} 4 \mathrm{H}_{2} \mathrm{O}$ & M & 2 & - & 2 & 2 & - & 2 & 2 & 2 \\
\hline $\mathrm{KNO}_{3}$ & $\mathrm{M}$ & 1 & - & 1 & - & 1 & 3 & 1 & 1 \\
\hline $\mathrm{K}_{2} \mathrm{SO}_{4}$ & M & 2 & 2 & 2 & - & 2 & 3 & - & 2 \\
\hline $\mathrm{MgSO}_{4}$ & $0,5 \mathrm{M}$ & 2.5 & 2.5 & 2.5 & 2.5 & 2.5 & - & - & 2,5 \\
\hline$\left(\mathrm{NH}_{4}\right)_{2} \mathrm{SO}_{4}$ & $\mathrm{M}$ & 1.5 & - & 1.5 & 2 & 2.5 & 2 & - & 1,5 \\
\hline $\mathrm{CaSO}_{4} \cdot 2 \mathrm{H}_{2} \mathrm{O}$ & $0,01 \mathrm{M}$ & - & 200 & - & - & - & - & - & - \\
\hline $\mathrm{KH}_{2} \mathrm{PO}_{4}$ & M & - & 1 & - & - & - & 1 & 1 & - \\
\hline $\mathrm{Mg}\left(\mathrm{NO}_{3}\right)_{2}$ & $0,5 \mathrm{M}$ & - & - & - & - & - & - & 2.5 & - \\
\hline $\mathrm{NaNO}_{3}$ & $\mathrm{M}$ & - & - & 1 & - & - & - & - & - \\
\hline SOL.A* & & 1 & 1 & 1 & 1 & 1 & 1 & 1 & $a-B$ \\
\hline SOL.Fe-EDTA** & & 1 & 1 & 1 & 1 & 1 & 1 & 1 & 1 \\
\hline
\end{tabular}

Fonte: Bolle-Jones (1954).

*Composição da solução A: 141,2 mg de $\mathrm{H}_{3} \mathrm{BO}_{3} ; 1.750 \mathrm{mg}$ de $\mathrm{MnSO}_{4} ; 250 \mathrm{mg}$ de $\mathrm{CuSO}_{4} 5 \mathrm{H}_{2} \mathrm{O} ; 43,1 \mathrm{mg}$ de $\mathrm{MoO}_{3}$; 287 mg de $\mathrm{ZnSO} 7 \mathrm{H} \mathrm{O}_{2}$, por litro de solução. **Composição da solução de Fe-EDTA: $26,1 \mathrm{~g}$ de $\mathrm{Na}_{2}$ - EDTA; 89,2g de NaOH N e 24g de FeSO $7 \mathrm{H}_{2} \mathrm{O}$, por litro de solução.

foram submetidas aos tratamentos completo e de omissão dos macronutrientes, com solução nutritiva diluída 1:1, com pH de 5,5. A solução nutritiva foi fornecida por percolação nos vasos de plástico, com capacidade para 3 L e renovada a cada 15 dias. Diariamente, observava-se o nível de solução nos frascos coletores, completando-os para $1 \mathrm{~L}$ por planta, quando necessário, com adição de água destilada. Quando os sintomas de deficiência, referentes a cada nutriente, se apresentaram bem definidos, as plantas foram coletadas e submetidas à lavagem com água destilada. Cada planta foi dividida em folhas, caule e raízes e colocadas para secar em estufa com circulação forçada de ar a $70^{\circ} \mathrm{C}$, até atingirem peso constante. Obtido o peso da matéria seca, procedeu-se à moagem do material em moinho tipo Willey, para análise química de tecido vegetal. Para o cálculo do crescimento relativo (CR), utilizou-se a fórmula:

$$
\mathrm{CR}(\%)=(\text { M.S.O.N/M.S.T.C. }) \times 100
$$

onde: $\quad$ M.S.O.N. = matéria seca da planta inteira obtida em cada omissão de nutriente;

M.S.T.C $=$ matéria seca total obtida no tratamento completo

Foram determinadas as concentrações de N, P, K, Ca, Mg, S e B nas folhas, caules e raízes, segundo metodologia descrita por Möller et al. (1997). Em função dos dados de matéria seca da planta e da concentração dos nutrientes, foram calculados os dados do acúmulo de nutrientes. Os dados obtidos foram analisados estatisticamente pelo programa de computador software - sas (sistema de análise estatística), para análise de variância, teste $\mathrm{F}$, e, obtida a significância, realizou-se o teste de Tukey, ao nível de $5 \%$ de probabilidade para a comparação de médias entre os tratamentos, em cada variável.

\section{RESULTADOSEDISCUSSÃO}

\section{Sintomas visuais de deficiências de macronutrientes e do boro}

\section{Nitrogênio}

Os sintomas de deficiência de nitrogênio surgiram 57 dias após o início dos tratamentos, as folhas mais velhas apresentaram coloração verdeamarelada e com a intensidade da deficiência, as lâminas foliares ficaram totalmente amareladas. Essa coloração, segundo Malavolta et al. (1997), está associada com a menor produção de clorofila e com modificações na forma dos cloroplastos.

\section{Fósforo}

Os sintomas de deficiência de fósforo surgiram 250 dias após iniciados os tratamentos, porém não se mostraram bem definidos. As folhas velhas apresentaram necrose no ápice e o tamanho reduzido, quando comparadas ao tratamento completo. A necrose no ápice caracteriza-se pela morte de tecidos, provavelmente em decorrência da perda de seletividade de membranas de plantas deficientes em fósforo, considerando que essas membranas são estruturas complexas, constituídas de proteínas e lipídios, nas quais este último componente é formado de fosfolipídios. De acordo com Mengel \& Kirkby (1987), plantas com deficiência em fósforo têm o seu crescimento retardado, por terem vários processos afetados, como a síntese de proteínas e a de ácidos nucléicos.

\section{Potássio}

Os sintomas de deficiência de potássio surgiram 139 dias após o início dos tratamentos, e se caracterizaram, inicialmente, com pequenas necroses nos bordos e nos ápices das folhas mais velhas, as quais, com a intensidade da deficiência, evoluíram e formaram grandes manchas necróticas no limbo foliar. Em plantas com deficiência em potássio, os compostos nitrogenados solúveis, inclusive as aminas putrescinas e agmatina, muitas vezes, se acumulam, sendo a última, provavelmente, responsável pelas manchas necróticas que aparecem nas folhas deficientes nesse nutriente (Epstein, 1975).

\section{Cálcio}

As plantas deficientes de cálcio apresentaram as folhas novas retorcidas, enroladas para a sua face ventral, ocorrendo essas características após 236 dias após iniciados os tratamentos. A falta de cálcio é caracterizada pela redução do crescimento de tecidos meristemáticos, sendo observado primeiro nas extremidades em crescimento e folhas mais jovens (Mengel \& Kirkby, 1987). No processo metabólico, o cálcio afeta a atividade de hormônio e de enzimas, como os que regulam a senescência e a abscisão das folhas e frutos (Malavolta, 1980; Marschner, 1995).

\section{Magnésio}

Os sintomas característicos de deficiência de magnésio em plantas de camucamuzeiro surgiram aos 139 dias após o início dos tratamentos, com clorose ao longo dos bordos das folhas mais velhas e amarelecimento entre as nervuras secundárias; com a intensidade da deficiência, ocorreu necrose. O magnésio é muito móvel no floema e, portanto, redistribui-se facilmente das folhas e tecidos mais velhos para regiões de maiores exigências, como os meristemas e órgãos de reserva (Epstein, 1975).

\section{Enxofre}

Os sintomas de deficiência de enxofre em plantas de camucamuzeiro foram os primeiros a se manifestarem, ou seja, após 30 dias de iniciados os tratamentos, tendo as folhas novas se apresentado com uma coloração verde-clara; com a intensidade da carência, ocorreu clorose entre as nervuras secundárias ao longo dos bordos das folhas. Em folhas verdes, a maioria das proteínas está localizada nos cloroplastos e nas moléculas da clorofila. Portanto, plantas deficientes em enxofre apresentam menor teor de clorofila e, conseqüentemente, uma coloração verde-pálida.

\section{Boro}

Os sintomas de deficiência de boro em plantas de camucamuzeiro manifestaram-se 50 dias depois de iniciados os tratamentos. As folhas novas se apresentaram retorcidas, atrofiadas, pequenas e grossas, e, com a intensidade dos sintomas, ocorreu morte do meristema apical do caule. Isso está relacionado, segundo Epstein (1975), ao ácido ribonucléico - 
TABELA 2 - Produção de matéria seca das folhas, caule, raízes e total (g/planta) de camucamuzeiro, crescimento relativo (CR \%) e relação parte aérea/ raiz (PA/R), em função dos tratamentos.

\begin{tabular}{|c|c|c|c|c|c|c|}
\hline Tratamento & Folhas & Caule & Raízes & Total & $\mathrm{CR}$ & $\mathrm{PA} / \mathrm{R}$ \\
\hline Completo & $14,75 \mathrm{a}$ & $48,69 \mathrm{a}$ & $32,73 \mathrm{a}$ & $96,17 \mathrm{a}$ & 100 & 1,9 \\
\hline Omissão de N & $2,47 \mathrm{e}$ & $7,11 \mathrm{e}$ & $6,65 \mathrm{c}$ & $16,24 \mathrm{e}$ & 16 & 1,4 \\
\hline Omissão de $\mathrm{P}$ & $15,82 \mathrm{a}$ & $55,01 \mathrm{a}$ & $29,45 \mathrm{a}$ & $100,29 \mathrm{a}$ & 104 & 2,4 \\
\hline Omissão de K & $5,44 \mathrm{~d}$ & $12,03 \mathrm{de}$ & $7,91 \mathrm{bc}$ & $25,38 \mathrm{~d}$ & 26 & 2,2 \\
\hline Omissão de $\mathrm{Ca}$ & $7,70 \mathrm{bc}$ & $24,93 \mathrm{~b}$ & $11,59 \mathrm{~b}$ & $44,22 \mathrm{~b}$ & 45 & 2,8 \\
\hline Omissão de $\mathrm{Mg}$ & $8,63 \mathrm{~b}$ & $17,25 \mathrm{~cd}$ & $10,49 \mathrm{bc}$ & $36,38 \mathrm{c}$ & 37 & 2,4 \\
\hline Omissão de S & $7,64 \mathrm{bc}$ & $19,80 \mathrm{bc}$ & 8,22 bc & $35,67 \mathrm{c}$ & 37 & 3,3 \\
\hline Omissão de B & $6,10 \mathrm{~cd}$ & $14,29 \mathrm{~cd}$ & $8,07 \mathrm{bc}$ & $28,46 \mathrm{~d}$ & 29 & 2,5 \\
\hline CV (\%) & 8,19 & 11,07 & 13,84 & 5,71 & & \\
\hline DMS & 1,64 & 6,45 & 4,66 & 6,40 & & \\
\hline
\end{tabular}

*Médias seguidas pela mesma letra nas colunas não diferem entre si, ao nível de 5\% de probabilidade, pelo teste de Tukey.

RNA. O boro se acumula nas folhas mais velhas, com teores mais altos nas pontas e margens, e o transporte desse micronutriente ocorre via transpiração, o que explicaria o fato de os sintomas de deficiência se manifestarem nos pontos de crescimento (Jones Jr., 1970). A deficiência de boro prejudica o transporte e a ação dos reguladores de crescimento, além de provocar distúrbios no desenvolvimento da planta, em razão do aumento do nível do ácido indolacético, redução da síntese de proteínas, dificuldade de formação da parede celular e no transporte de produtos da fotossíntese, propiciando o acúmulo de compostos fenólicos no vegetal (Melo \& Lemos, 1991). Algumas plantas da Amazônia Oriental, destacandose o dendezeiro e o coqueiro, são sensíveis à deficiência de boro (Viégas $\&$ Botelho, 2000).

Efeito dos tratamentos sobre a produção de matéria seca e crescimento relativo em plantas de camucamuzeiro

Com exceção do tratamento com omissão de fósforo, os demais tratamentos limitaram a produção de matéria seca nas folhas, no caule, nas raízes e total, quando comparados ao completo (Tabela 2). Fato semelhante foi observado por Viégas et al. (1998) em plantas de quina (Quassia amara L.).Provavelmente, a não-exteriorização de efeitos deletérios sobre a produção de matéria seca, em função da omissão de fósforo observado neste trabalho, se deva às quantidades absorvidas desse nutriente durante a fase de aclimatação das plantas na solução nutritiva. Os tratamentos que mais afetaram a produção de matéria seca total foram as omissões individuais de nitrogênio, potássio e boro, quando comparados ao tratamento completo. Constatou-se que o crescimento relativo (CR) obedeceu à seguinte ordem decrescente, em relação aos tratamentos: $\mathrm{P}>$ Completo $>\mathrm{Ca}>\mathrm{Mg}=\mathrm{S}>\mathrm{B}>\mathrm{K}>\mathrm{N}$. Deduz-se, dessa maneira, que o desenvolvimento da planta, durante o período experimental, não foi afetado pela omissão de fósforo, foi menos afetado pela omissão de $\mathrm{Ca}$, com redução de $55 \%$ da matéria seca, e mais afetado pela omissão de nitrogênio, com redução de $84 \%$ da matéria seca. Essa limitação do crescimento, resultante da omissão de nitrogênio, também foi constatada em plantas de

TABELA 3 - Teores de macronutrientes $\left(\mathrm{g} \mathrm{kg}^{-1}\right)$ e de boro $\left(\mathrm{mg} \mathrm{kg}^{-1}\right)$ nas folhas, caules e raízes de camucamuzeiro, em função dos tratamentos.

\begin{tabular}{|c|c|c|c|c|c|c|c|}
\hline Tratamentos & $\mathrm{N}$ & $\mathrm{P}$ & $\mathrm{K}$ & $\mathrm{Ca}$ & $\mathrm{Mg}$ & $\mathrm{S}$ & $\mathrm{B}$ \\
\hline & & & Folhas & & & & \\
\hline Completo & $17,70 \mathrm{a}$ & $1,45 \mathrm{~b}$ & $5,60 \mathrm{~b}$ & $10,87 \mathrm{a}$ & $2,17 \mathrm{~cd}$ & $2,62 \mathrm{a}$ & $9,05 \mathrm{a}$ \\
\hline Omissão de $\mathrm{N}$ & $7,17 \mathrm{c}$ & $1,12 \mathrm{bc}$ & $4,30 \mathrm{c}$ & $6,32 \mathrm{~cd}$ & $2,77 \mathrm{bcd}$ & $1,42 \mathrm{~b}$ & $5,42 \mathrm{bc}$ \\
\hline Omissão de P & $17,67 \mathrm{a}$ & $0,90 \mathrm{c}$ & $4,17 \mathrm{c}$ & $10,25 \mathrm{ab}$ & $3,85 \mathrm{~b}$ & $2,47 \mathrm{a}$ & $4,27 \mathrm{~cd}$ \\
\hline Omissão de K & $17,85 \mathrm{a}$ & $2,27 \mathrm{a}$ & $1,67 \mathrm{~d}$ & $7,95 \mathrm{bc}$ & $5,22 \mathrm{a}$ & $2,45 \mathrm{a}$ & $8,25 \mathrm{a}$ \\
\hline Omissão de Ca & $16,20 \mathrm{a}$ & $1,22 \mathrm{bc}$ & $5,02 \mathrm{bc}$ & $6,00 \mathrm{~cd}$ & $3,55 \mathrm{~b}$ & $2,52 \mathrm{a}$ & $4,10 \mathrm{~d}$ \\
\hline Omissão de Mg & $18,72 \mathrm{a}$ & $1,50 \mathrm{~b}$ & $8,80 \mathrm{a}$ & $5,47 \mathrm{~d}$ & $0,70 \mathrm{e}$ & $2,72 \mathrm{a}$ & $6,05 \mathrm{~b}$ \\
\hline Omissão de S & $12,82 \mathrm{~b}$ & $1,17 \mathrm{bc}$ & $8,55 \mathrm{a}$ & $6,15 \mathrm{~cd}$ & $1,75 \mathrm{de}$ & $0,97 \mathrm{c}$ & $2,00 \mathrm{e}$ \\
\hline Omissão de B & $18,90 \mathrm{a}$ & $1,25 \mathrm{bc}$ & $4,92 \mathrm{bc}$ & $7,40 \mathrm{~cd}$ & $3,17 \mathrm{bc}$ & $2,30 \mathrm{a}$ & $1,60 \mathrm{e}$ \\
\hline CV (\%) & 7,95 & 13,64 & 9,97 & 13,18 & 18,64 & 8,52 & 10,04 \\
\hline DMS & 2,96 & 0,43 & 1,25 & 2,33 & 1,26 & 0,43 & 1,19 \\
\hline & & & Caule & & & & \\
\hline Completo & $9,85 \mathrm{a}$ & $1,35 \mathrm{c}$ & $8,10 \mathrm{bc}$ & $4,62 \mathrm{a}$ & $3,17 \mathrm{a}$ & $2,42 \mathrm{a}$ & $2,37 \mathrm{ab}$ \\
\hline Omissão de $\mathrm{N}$ & $1,72 \mathrm{f}$ & $1,27 \mathrm{c}$ & $7,40 \mathrm{c}$ & $4,95 \mathrm{a}$ & $1,50 \mathrm{bc}$ & $1,55 \mathrm{~b}$ & $1,42 \mathrm{de}$ \\
\hline Omissão de $\mathrm{P}$ & $5,25 \mathrm{de}$ & $0,62 \mathrm{~d}$ & $8,32 \mathrm{bc}$ & $4,80 \mathrm{a}$ & $1,57 \mathrm{bc}$ & $2,55 \mathrm{a}$ & $1,90 \mathrm{c}$ \\
\hline Omissão de K & $6,37 \mathrm{~cd}$ & $1,72 \mathrm{ab}$ & $2,47 \mathrm{~d}$ & $3,67 \mathrm{bc}$ & $1,92 \mathrm{~b}$ & $1,85 \mathrm{~b}$ & $1,90 \mathrm{c}$ \\
\hline Omissão de $\mathrm{Ca}$ & $8,37 \mathrm{~b}$ & $1,52 \mathrm{bc}$ & $9,67 \mathrm{a}$ & $2,47 \mathrm{~d}$ & $1,47 \mathrm{bc}$ & $2,47 \mathrm{a}$ & $2,00 \mathrm{bc}$ \\
\hline Omissão de Mg & $7,50 \mathrm{bc}$ & $2,05 \mathrm{a}$ & $8,67 \mathrm{ab}$ & $3,40 \mathrm{c}$ & $0,22 \mathrm{~d}$ & $1,87 \mathrm{~b}$ & $2,47 \mathrm{a}$ \\
\hline Omissão de $\mathrm{S}$ & $4,07 \mathrm{e}$ & $1,42 \mathrm{bc}$ & $9,87 \mathrm{a}$ & $3,30 \mathrm{~cd}$ & $1,10 \mathrm{c}$ & $0,30 \mathrm{c}$ & $1,25 \mathrm{e}$ \\
\hline Omissão de B & $6,90 \mathrm{c}$ & $1,22 \mathrm{c}$ & $7,87 \mathrm{bc}$ & $4,35 \mathrm{ab}$ & $1,45 \mathrm{bc}$ & $1,85 \mathrm{~b}$ & $1,72 \mathrm{~cd}$ \\
\hline CV (\%) & 9,55 & 10,95 & 6,78 & 8,93 & 16,97 & 10,65 & 8,9 \\
\hline DMS & 1,39 & 0,35 & 1,23 & 0,82 & 0,61 & 0,46 & 0,39 \\
\hline & & & Laízes & & & & \\
\hline Completo & $12,67 \mathrm{~b}$ & $1,77 \mathrm{~b}$ & $11,45 \mathrm{a}$ & $6,40 \mathrm{a}$ & $4,40 \mathrm{a}$ & $3,60 \mathrm{a}$ & $2,60 \mathrm{a}$ \\
\hline Omissão de $\mathrm{N}$ & $3,77 \mathrm{e}$ & $1,32 \mathrm{~b}$ & $9,30 \mathrm{abc}$ & $6,50 \mathrm{a}$ & $3,27 \mathrm{~b}$ & $2.80 \mathrm{~b}$ & $2,22 \mathrm{abc}$ \\
\hline Omissão de P & $8,90 \mathrm{c}$ & $0,47 \mathrm{c}$ & $10,07 \mathrm{abc}$ & $7,12 \mathrm{a}$ & $3,37 \mathrm{~b}$ & $3,82 \mathrm{a}$ & $2,30 \mathrm{ab}$ \\
\hline Omissão de K & $9,20 \mathrm{c}$ & $1,47 \mathrm{~b}$ & $1,67 \mathrm{~d}$ & $4,67 \mathrm{~b}$ & $2,50 \mathrm{c}$ & $2,57 \mathrm{~b}$ & $1,42 \mathrm{de}$ \\
\hline Omissão de $\mathrm{Ca}$ & $14,55 \mathrm{a}$ & $2,07 \mathrm{ab}$ & $8,67 \mathrm{bc}$ & $2,37 \mathrm{c}$ & $1,67 \mathrm{~d}$ & $3,50 \mathrm{a}$ & $2,50 \mathrm{ab}$ \\
\hline Omissão de Mg & $11,35 \mathrm{~b}$ & $2,75 \mathrm{a}$ & $11,05 \mathrm{ab}$ & $5,57 \mathrm{ab}$ & $0,47 \mathrm{e}$ & $2,95 \mathrm{~b}$ & $1,65 \mathrm{cde}$ \\
\hline Omissão de $\mathrm{S}$ & $7,27 \mathrm{~d}$ & $1,57 \mathrm{~b}$ & $7,92 \mathrm{c}$ & $6,07 a b$ & $1,30 \mathrm{~d}$ & $0,55 \mathrm{c}$ & $1,22 \mathrm{e}$ \\
\hline Omissão de B & $9,30 \mathrm{c}$ & $1,37 \mathrm{~b}$ & $8,00 \mathrm{c}$ & $5,67 \mathrm{ab}$ & $2,47 \mathrm{c}$ & $2,90 \mathrm{~b}$ & $2,00 \mathrm{bcd}$ \\
\hline CV (\%) & 7,07 & 22,28 & 12,45 & 12,84 & 11,96 & 7,59 & 12,35 \\
\hline DMS & 1,59 & 0,83 & 2,48 & 1,67 & 0,68 & 0,50 & 0,57 \\
\hline
\end{tabular}

*Médias seguidas pela mesma letra nas colunas não diferem entre si, ao nível de $5 \%$ de probabilidade, pelo teste de Tukey. 
juta por Viégas et al. (1992), de malva por Fasabi (1996) e de urucum por Haag et al. (1988). Tal efeito é justificado em razão de o nitrogênio ser constituinte dos aminoácidos e, conseqüentemente, de proteínas, e quando ocorre a deficiência de nitrogênio, há alteração no metabolismo vegetal, causando prejuízos ao desenvolvimento das plantas. Os resultados também evidenciaram que a deficiência de enxofre apresentou a relação parte aérea/raiz com maior quociente, portanto com menor quantidade de matéria seca de raízes. Isso pode se explicado pela diminuição na partição de carboidratos para a raiz, provavelmente devido à diminuição do teor de sacarose das raízes de plantas deficientes em enxofre.

Efeito dos tratamentos sobre os teores de macronutrientes e de boro nas folhas, caule e raízes de plantas de camucamuzeiro

Os resultados dos teores de macronutrientes correspondentes a cada tratamento são apresentados na Tabela 3. Constata-se que as omissões isoladas de nitrogênio, fósforo, potássio, cálcio, magnésio, enxofre e boro reduziram os teores desses nutrientes em todas as partes da planta, quando comparados com o tratamento completo. $\mathrm{O}$ fato foi observado em plantas de quina por Viégas et al. (1998), com exceção da omissão do cálcio, que não reduziu o teor desse nutriente nas folhas e raízes

As concentrações de macronutrientes das plantas do tratamento completo obedeceram à seguinte ordem nas folhas: $\mathrm{N}>\mathrm{Ca}>\mathrm{K}>\mathrm{S}>\mathrm{Mg}>\mathrm{P}$; no caule e raízes, $\mathrm{N}>\mathrm{K}>\mathrm{Ca}>\mathrm{Mg}>\mathrm{S}>\mathrm{P}$. Com base nos teores dos macronutrientes $\left(\mathrm{g} \mathrm{kg}^{-1}\right.$, ) e do micronutriente boro $\left(\mathrm{mg} \mathrm{kg}^{-1}\right)$ nas folhas de camucamuzeiro, infere-se em uma primeira aproximação dos valores normais (completo) sendo : $\mathrm{N}$ de 16,9 a 18,2; $\mathrm{P}$ de1,2 a 1,9; K de 5,2 a 6,0; Ca de 9,9 a 11,7; Mg de 1,4 a 3,6; $\mathrm{S}$ de 2,4 a 2,8; B de 8,4 a 9,5 e do deficiente (omissão) , $\mathrm{N}$ de 6,5 a 7,$9 ; \mathrm{P}=0,9 ; \mathrm{K}=1,7 ;$ Ca de 5,4 a 6,$5 ; \mathrm{Mg}=0,7 ; \mathrm{S}$ de 0,7 a 1,2 e $\mathrm{B}$ de 1,1 a 1,9 .

As faixas dos teores foliares adequadas, como valores de referência sugeridas nesta pesquisa de fósforo, cálcio, magnésio e enxofre, são compatíveis com os teores considerados adequados para a goiabeira, mesma família do camucamuzeiro, a partir do terceiro ano de idade, cultivar Paluma (Natale et al., 1996). Todavia, o limite superior de $6 \mathrm{~g} \mathrm{~kg}^{-1} \mathrm{de} \mathrm{K} \mathrm{em}$ folhas de camucamuzeiro, obtido nesta pesquisa, é inferior em cerca de $130 \%$ ao determinado em folhas de goiabeira por Natale et al. (1996), indicando ser o camucamuzeiro menos exigente em potássio do que a

TABELA 4 - Acúmulo de macronutrientes $\left(\mathrm{g} \mathrm{kg}^{-1}\right)$ e de boro $\left(\mathrm{mg} \mathrm{kg}^{-1}\right)$ nas folhas, caule, raízes e total de camucamuzeiro, em função dos tratamentos.

\begin{tabular}{|c|c|c|c|c|c|c|c|}
\hline Tratamentos & $\mathrm{N}$ & $\mathrm{P}$ & $\mathrm{K}$ & $\mathrm{Ca}$ & $\mathrm{Mg}$ & $\mathrm{S}$ & $\mathrm{B}$ \\
\hline \multicolumn{8}{|c|}{ Folhas } \\
\hline Completo & $261,23 a$ & $21,32 \mathrm{a}$ & $82,54 a$ & $150,33 a$ & $32,75 b$ & $38,73 a$ & $133,74 a$ \\
\hline Omissão de N & $17,72 \mathrm{~d}$ & $2,77 \mathrm{e}$ & $10,53 \mathrm{~d}$ & $15,65 \mathrm{c}$ & $6,89 \mathrm{~d}$ & $3,52 \mathrm{e}$ & $13,45 \mathrm{ef}$ \\
\hline Omissão de P & $279,64 a$ & $14,24 b$ & $66,03 \mathrm{ab}$ & $162,28 \mathrm{a}$ & $60,97 \mathrm{a}$ & $39,19 \mathrm{a}$ & $67,70 \mathrm{~b}$ \\
\hline Omissão de K & $96,89 \mathrm{c}$ & $12,36 \mathrm{bcd}$ & $9,12 \mathrm{~d}$ & $43,20 b c$ & $28,30 \mathrm{bc}$ & $13,31 \mathrm{~cd}$ & $44,85 \mathrm{~cd}$ \\
\hline Omissão de $\mathrm{Ca}$ & $125,01 \mathrm{c}$ & $9,47 \mathrm{bcd}$ & $38,59 \mathrm{c}$ & $46,38 b$ & $27,37 \mathrm{bc}$ & $19,48 b c$ & $31,25 \mathrm{de}$ \\
\hline Omissão de $\mathrm{Mg}$ & $160,70 b$ & $12,96 b c$ & $76,30 \mathrm{ab}$ & $48,26 \mathrm{~b}$ & $6,04 d$ & $23,50 b$ & $51,89 b c$ \\
\hline Omissão de S & $97,98 \mathrm{c}$ & $8,99 \mathrm{~cd}$ & $65,41 b$ & $47,01 \mathrm{~b}$ & $13,37 d$ & $7,44 \mathrm{de}$ & $15,30 \mathrm{ef}$ \\
\hline Omissão de B & $114,98 \mathrm{c}$ & $7,63 \mathrm{de}$ & $29,99 \mathrm{c}$ & $45,34 \mathrm{~b}$ & $19,46 \mathrm{bcd}$ & $14,03 \mathrm{~cd}$ & $9,76 \mathrm{f}$ \\
\hline CV (\%) & 10,33 & 18,93 & 15,36 & 17,33 & 28,89 & 14,15 & 17,10 \\
\hline \multirow[t]{2}{*}{ DMS } & 34,92 & 4,97 & 17,02 & 29,94 & 16,50 & 6,59 & 19,44 \\
\hline & & & Caule & & & & \\
\hline Completo & $479,35 \mathrm{a}$ & $65,95 \mathrm{a}$ & $394,53 b$ & $225,04 a$ & $154,91 \mathrm{a}$ & $118,08 b$ & $115,62 \mathrm{a}$ \\
\hline Omissão de N & $12,6 f$ & $9,09 \mathrm{e}$ & $52,54 \mathrm{f}$ & $35,18 \mathrm{~b}$ & $10,54 d$ & $11,03 \mathrm{ef}$ & $10,14 d$ \\
\hline Omissão de P & $288,6 b$ & $34,33 b c$ & $457,98 \mathrm{a}$ & $264,44 \mathrm{a}$ & $86,59 b$ & $140,21 \mathrm{a}$ & $104,49 a$ \\
\hline Omissão de K & $72,9 \mathrm{e}$ & 20,52 cde & $29,50 \mathrm{f}$ & $43,31 \mathrm{~b}$ & $22,52 \mathrm{~cd}$ & $21,85 \mathrm{de}$ & $21,86 \mathrm{~cd}$ \\
\hline Omissão de Ca & $209,33 c$ & $37,93 \mathrm{~b}$ & $241,56 \mathrm{c}$ & $61,92 b$ & $40,36 c$ & $61,79 c$ & $49,85 b$ \\
\hline Omissão de Mg & $129,53 d$ & $35,51 \mathrm{~b}$ & $149,58 \mathrm{de}$ & $58,63 \mathrm{~b}$ & $3,83 \mathrm{~d}$ & $32,16 \mathrm{~d}$ & $42,73 b$ \\
\hline Omissão de S & $80,72 \mathrm{de}$ & $28,13 \mathrm{bcd}$ & $194,14 \mathrm{~cd}$ & $65,39 b$ & $21,72 \mathrm{~cd}$ & $5,95 f$ & $24,67 \mathrm{c}$ \\
\hline Omissão de B & $100,60 \mathrm{de}$ & $17,39 \mathrm{de}$ & $113,18 \mathrm{e}$ & $62,64 b$ & $20,95 \mathrm{~cd}$ & $26,20 \mathrm{de}$ & $24,75 \mathrm{c}$ \\
\hline CV $(\%)$ & 13,53 & 19,16 & 12,36 & 17,33 & 22,41 & 12,61 & 12,03 \\
\hline \multirow[t]{2}{*}{ DMS } & 54,41 & 13,96 & 59,14 & 41,34 & 23,71 & 15,40 & 13,89 \\
\hline & & & Raízes & & & & \\
\hline Completo & $418,21 \mathrm{a}$ & $57,01 \mathrm{a}$ & $374,87 a$ & $209,54 a$ & $143,67 a$ & $118,79 \mathrm{a}$ & $84,51 \mathrm{a}$ \\
\hline Omissão de N & $25,08 \mathrm{e}$ & $8,79 \mathrm{~d}$ & $62,19 \mathrm{~cd}$ & $43,09 \mathrm{~b}$ & $21,80 \mathrm{c}$ & $18,63 b c$ & $14,78 \mathrm{~cd}$ \\
\hline Omissão de P & $262,15 b$ & $13,99 \mathrm{~cd}$ & $296,89 b$ & $209,74 a$ & $99,37 b$ & $112,64 \mathrm{a}$ & $67,80 \mathrm{~b}$ \\
\hline Omissão de K & $72,89 \mathrm{de}$ & $11,71 d$ & $13,21 d$ & $36,83 b$ & $19,64 \mathrm{c}$ & $20,37 b c$ & $11,28 \mathrm{~d}$ \\
\hline Omissão de Ca & $168,95 \mathrm{c}$ & $23,89 \mathrm{bc}$ & $100,15 \mathrm{c}$ & $27,53 b$ & $19,36 \mathrm{c}$ & $40,65 b$ & $29,05 \mathrm{c}$ \\
\hline Omissão de $\mathrm{Mg}$ & $119,64 \mathrm{~cd}$ & $29,28 b$ & $116,19 \mathrm{c}$ & $58,73 b$ & $5,08 \mathrm{c}$ & $30,77 \mathrm{~b}$ & $17,15 \mathrm{~cd}$ \\
\hline Omissão de S & $60,07 \mathrm{de}$ & $12,96 \mathrm{~d}$ & $65,69 \mathrm{~cd}$ & $49,66 \mathrm{~b}$ & $10,68 \mathrm{c}$ & $4,52 \mathrm{c}$ & $10,11 \mathrm{~d}$ \\
\hline Omissão de B & $74,97 \mathrm{de}$ & $10,84 \mathrm{~d}$ & $64,32 \mathrm{~cd}$ & $46,87 \mathrm{~b}$ & $19,69 \mathrm{c}$ & $23,38 \mathrm{bc}$ & $16,11 \mathrm{~cd}$ \\
\hline CV (\%) & 25,66 & 20,80 & 21,56 & 21,23 & 19,60 & 22,59 & 21,39 \\
\hline \multirow[t]{2}{*}{ DMS } & 90,31 & 10,26 & 69,03 & 42,32 & 19,47 & 24,46 & 15,70 \\
\hline & & & Total & & & & \\
\hline Completo & $1158,79 a$ & $144,28 \mathrm{a}$ & $851,94 a$ & $594,91 \mathrm{a}$ & $331,34 \mathrm{a}$ & $275,53 a$ & $333,87 \mathrm{a}$ \\
\hline Omissão de N & $55,06 \mathrm{f}$ & $20,67 f$ & $125,26 \mathrm{~cd}$ & $93,92 \mathrm{c}$ & $39,24 \mathrm{de}$ & $32,19 \mathrm{e}$ & $38,37 \mathrm{e}$ \\
\hline Omissão de P & $830,64 b$ & $62,57 \mathrm{bcd}$ & $820,90 a$ & $636,47 a$ & $246,94 b$ & $292,05 a$ & $239,99 b$ \\
\hline Omissão de K & $242,57 \mathrm{e}$ & $44,69 \mathrm{de}$ & $51,75 d$ & $123,39 \mathrm{bc}$ & $70,48 \mathrm{~cd}$ & $55,53 d$ & $77,99 \mathrm{~d}$ \\
\hline Omissão de $\mathrm{Ca}$ & $503,29 \mathrm{c}$ & $71,23 b c$ & $380,38 b$ & $135,78 \mathrm{bc}$ & $87,10 \mathrm{c}$ & $121,92 b$ & $110,61 \mathrm{c}$ \\
\hline Omissão de Mg & $409,87 d$ & $77,76 b$ & $342,06 b$ & $165,63 \mathrm{~b}$ & $14,96 \mathrm{e}$ & $86,44 \mathrm{c}$ & $110,78 \mathrm{c}$ \\
\hline Omissão de S & $238,68 \mathrm{e}$ & $50,07 \mathrm{cde}$ & $326,37 b$ & $162,09 \mathrm{~b}$ & $45,78 \mathrm{de}$ & $17,92 \mathrm{e}$ & $50,09 \mathrm{e}$ \\
\hline Omissão de B & $290,55 \mathrm{e}$ & $35,88 \mathrm{ef}$ & $207,49 c$ & $153,86 \mathrm{bc}$ & $60,11 \mathrm{~cd}$ & $63,62 d$ & $50,72 \mathrm{e}$ \\
\hline$\overline{C V}(\%)$ & 7,07 & 15,20 & 9,60 & 10,82 & 14,14 & 7,43 & 8,85 \\
\hline DMS & 77,21 & 22,57 & 87,29 & 65,46 & 37,10 & 20,59 & 26,26 \\
\hline
\end{tabular}

Médias seguidas pela mesma letra nas colunas não diferem entre si, ao nível de $5 \%$ de probabilidade, pelo teste de Tukey. 
goiabeira. A faixa do teor foliar de 8,4 a 9,5 $\mathrm{mg} \mathrm{kg}^{-1} \mathrm{de} \mathrm{B}$, em camucamuzeiro, é muito baixa, quando comparada à faixa adequada de goiabeira de $20 \mathrm{a}$ $25 \mathrm{mg} \mathrm{kg}^{-1}$. Essa comparação entre os resultados conflitantes dos teores foliares de K e B de camucamuzeiro e os de goiabeira, indicados pelos citados autores, deve ser vista com cautela, pelo fato de terem sido obtidos em condições diferentes: casa de vegetação, utilizando mudas em solução nutritiva e plantas adultas em campo, respectivamente, e, principalmente, por serem espécies distintas e com exigências nutricionais diferentes.

Efeito dos tratamentos sobre o acúmulo de macronutrientes e de boro nas folhas, caule e raízes de plantas de camucamuzeiro.

A exemplo do ocorrido com os teores, as omissões isoladas dos macronutrientes e do boro limitaram o acúmulo de nitrogênio, fósforo, potássio, cálcio, magnésio, enxofre e boro nas folhas, caule, raízes e na planta inteira, quando comparados com o tratamento completo (Tabela 4). Esses resultados demonstram a importância desses nutrientes para o cultivo racional do camucamuzeiro. A ordem decrescente do acúmulo total de nutrientes, com base no tratamento completo, foi: $\mathrm{N}>\mathrm{K}>\mathrm{Ca}>\mathrm{S}>\mathrm{Mg}$ $>\mathrm{P}>\mathrm{B}$, mostrando que os nutrientes mais exigidos pelo camucamuzeiro foram nitrogênio e potássio, a exemplo da goiabeira (Salvador et al., 1999) e da gravioleira (Barbosa, 2000). O acúmulo total de 1.158,79 $\mathrm{mg} \mathrm{planta}^{-1} \mathrm{de}$ $\mathrm{N}$, obtido no tratamento completo, corresponde, sem considerar as perdas ocorridas no solo, a aproximadamente 2,6 g planta $^{-1}$ de uréia, valor que deve indicar a necessidade de adubação nitrogenada para plantas de camucamuzeiro com 310 dias de idade, tempo de duração do experimento. O acúmulo total de $851,94 \mathrm{mg} /$ planta de $\mathrm{K}$, também do tratamento completo, corresponde à quantidade de $1,7 \mathrm{~g}_{\text {planta }}{ }^{-1}$ de cloreto de potássio, enquanto o acumulado total de $144,28 \mathrm{mg} /$ planta de $P$ equivale a $0,7 \mathrm{~g} \mathrm{planta}^{-1} \mathrm{de}$ superfosfato triplo. Chama atenção, o acúmulo total de cálcio de 593,25 mg planta $^{-1}$, o terceiro nutriente mais absorvido pelo camucamuzeiro.

\section{CONCLUSÕES}

1. Com exceção da omissão de fósforo, os demais tratamentos limitam a produção de matéria seca das folhas, do caule, das raízes e total em plantas de camucamuzeiro, quando comparados ao tratamento completo.

2. As omissões individuais de nitrogênio, potássio e boro são as mais limitantes para a produção de matéria seca total em plantas de camucamuzeiro.

3. As omissões de N, P, K, Ca, Mg, S e B, na solução nutritiva, resultam em alterações morfológicas, traduzidas como sintomas característicos de deficiência nutricional de cada nutriente em camucamuzeiro.

4. As omissões de N, P, K, Mg, Ca, Mg, S e B resultam em redução dos teores e do acúmulo dos nutrientes nas diversas partes de plantas de camucamuzeiro.

\section{REFERÊNCIAS BIBLIOGRÁFICAS}

BARBOSA, Z. Produção de biomassa e acumulação de nutrientes pela gravioleira (Anona muricata L.) em condições de viveiro e de campo. 2000.61f.Dissertação (Mestrado em Fitotecnia) Universidade Federal do Ceará, Fortaleza, 2000.

BOLLE-JONES, E. W. Nutrition of (Hevea brasiliensis) II. Effects of nutrient deficiencies on growth, chlorophyll, rubber and contents of Tjirandji seedlings. Journal of Rubber Research Institute of Malaya, v. 14, p. 209, 1954.
EPSTEIN, E. Nutrição mineral de plantas, princípios e perspectivas. São Paulo: Universidade de São Paulo, 1975. 341p.

FASABI, J. A. V. Carências de macro e micronutrientes em plantas de malva (Urena lobata), variedade BR-01. 1996. 90p. Dissertação (Mestrado em Agronomia) - Faculdade de Ciências Agrárias do Pará, Belém, 1996.

HAAG, H. P.; DECHEN, A. R.; ROSOLEN, D. L. Carência de macronutrientes e de boro em plantas de urucu. Anais da Escola de Agricultura Luiz de Queiroz, Piracicaba, v.45, n.2, p. 423-431, 1988.

JONES Jr., J.B. Distribution of 15 elements in corn leaves. Communications in Soil Science and Plant Analysis, Monticello, New York, 1, p. 27-34,1970.

LUDWIG, M.M. A nova rainha da vitamina C: camu-camu saúde é vida. São Paulo:Azul, 1996. p.15-23.

MALAVOLTA, E. Elementos de nutrição mineral de plantas. São Paulo: Ceres, 1980.251p.

MALAVOLTA, E.; VITTI, G. C.; OLIVEIRA, S. A. Avaliação do estado nutricional das plantas: princípios e aplicações. Piracicaba: POTAFOS, 1997.319p.

MARSCHNER, H. Mineral nutrition of higher plants. London: Academic Press, 1995. 889p.

MELO, J.W.; LEMOS, E.G.M. Análise bioquímica de plantas.In: I SIMPÓSIO SOBRE MICRONUTRIENTES NAAGRICULTURA. Jaboticabal 1, 1988, Piracicaba. Anais...Piracicaba: POTAFOS/CNPq, 1991.p. $310-331$.

MENGEL, K.; KIRKBY, E.A. Principles of plant nutrition. Bern: Intern. Potash Institute, 1987.687p.

MOLLER, M.R.F.; VIÉGAS, I. de J.M.; MATOS, A. de O.; PARRY, M.M. Análise de tecido vegetal: manual de laboratório. Belém: Embrapa Amazônia Oriental, 1997.32p. (Documento, 92).

NATALE, W.; COUTINHO, E.LM.; BOARETO, A.E.; PEREIRA, F.M. Goiabeira : calagem e adubação. Jaboticabal : FUNEP, 1996. 22p.

RIBEIRO, S.I.; MOTA, M.G. da C.;CORRÊA, M.R.P.Recomendações para o cultivo do camucamuzeiro no Estado do Pará.Belém: Embrapa Amazônia Oriental, 2002. 9p. (Embrapa Amazônia Oriental, Circular Técnica, 31).

SALVADOR, J.O.; MOREIRA, A. ; MURAOKA, T. Efeito da omissão combinada de N, P, K e S nos teores foliares de macronutrientes em mudas de goiabeira. Scientia Agrícola, Piracicaba, v.56, n.2, p. 501 507. 1999.

VIÉGAS, I. de J. M.; BOTELHO, S. M.. Nutrição e adubação do dendezeiro. Organizado por VIEGAS, I. de J. M.; MÜLLER, A. A. A cultura do dendezeiro na Amazônia brasileira. Belém, 2000, v.1, p.229273.

VIÉGAS, I. de J. M.; CARVALHO, J. G. de; ROCHA NETO, O. G. da.; SANTIAGO, E. A. de. Carência de macronutrientes em plantas de quina. Belém: Embrapa-CPATU, 1998.31p. (Boletim de Pesquisa,192).

VIÉGAS, I. de J. M.; HAAG, H. P.; SILVA, F da S.; MONTEIRO, F. A. Carência de macronutrientes e de boro em plantas de juta (Corchorus capsularis L.), variedade roxa. Belém: Embrapa-CPATU, 1992. 24p. (Boletim de Pesquisa, 138).

YUYAMA, L.K.O.; AGUIAR, J.P.L.; YUYAMA, K.; LOPES,T.M.; FÁVARO, D.I.T.; BERGL, P.C.P.; VASCONCELLOS, M.B.A.Teores de elementos minerais em algumas populações de camu-camu.Acta Amazônica, Manaus, INPA, 33(4), p. 549-554, 2003. 